\title{
Los métodos geofísicos como herramienta de mejora para el conocimiento y aprovechamiento de los yacimientos de roca ornamental
}

\author{
A. Espín de Gea, M. Reyes Urquiza y A. Gil Abellán \\ Departamento de Geología, Topografía y Geofísica, Centro Tecnológico del Mármol, Piedra y Materiales, \\ Polígono Industrial El Matadero, Carretera de Murcia s/n, 30430 Cehegín (Murcia) \\ antonio.espin@ctmarmol.es \\ monica.reyes@ctmarmol.es \\ antonio.gil@ctmarmol.es
}

RESUMEN

El sector de la roca ornamental, y más concretamente la investigación de los yacimientos, es poco dado a la incorporación de nuevas tecnologías. El Centro Tecnológico del Mármol (CTM) avanza en este sector con la utilización de técnicas geofísicas capaces de obtener información del subsuelo. Existen todavía pocos afloramientos y canteras activas donde se hayan aplicado técnicas geofísicas de manera conjunta y con la intensidad suficiente como para obtener un modelo tridimensional de la zona estudiada.

La aplicación de diversas metodologías geofísicas, y más concretamente georadar, tomografía eléctrica y sísmica de refracción, a la hora de realizar la investigación de un yacimiento o afloramiento, constituyen herramientas poco agresivas con el medioambiente, que permiten conocer las características de un yacimiento de forma real antes de realizar labores más agresivas, como es el caso de la ejecución de sondeos y calicatas a las cuales posteriormente puede complementar. Se puede tener así una idea previa muy aproximada de la calidad del yacimiento que permite una optimización de los recursos, tanto económicos como medioambientales.

Mediante varios trabajos Ilevados a cabo por el CTM, se ilustran tanto los beneficios aportados por la aplicación de estas técnicas geofísicas, como sus puntos débiles dentro de las características de las explotaciones y afloramientos de roca ornamental.

Palabras clave: geofísica, resistividad, roca ornamental, sondeo, yacimiento.

\section{Geophysical methods as a tool for improving our knowledge of ornamental rock deposits and their exploitation}

\begin{abstract}
The ornamental rock sector, particularly the investigation of deposits, is not readily given to the incorporation of new technologies. The Marble Technology Center (CTM) has made advances in this sector with the implementation of geophysical techniques able to obtain subsurface information. There are still a few outcrops and active quarries where joint geophysical techniques have been applied sufficiently to obtain a three-dimensional model of the study area.

The application of geophysics and more specifically, ground penetrating radar, electrical tomography and seismic refraction, when conducting research of a reservoir or outcrop, is only slightly aggressive to the environment, allowing a real knowledge of the characteristics before beginning the more aggressive work, as is the case when conducting surveys and test pits which can subsequently complement the data obtained. This makes it possible to gain a good idea of the quality of the reservoir thus allowing optimization of resources, both financial and environmental beforehand.

Several studies conducted by the CTM illustrate the benefits brought by the application of these geophysical techniques as well as the weaknesses within the characteristics of the extraction sites and ornamental rock outcrops.
\end{abstract}

Keywords: borehole, deposit, geophysics, ornamental stone, resistivity. 
Espín de Gea, A., et al., 2017. Los métodos geofísicos como herramienta de mejora para... Boletín Geológico y Minero, 128 (2): $363-378$

\section{ABRIDGED ENGLISH VERSION}

\section{Introduction and methods}

The Marble Technology Center carries out different research methods to facilitate the implementation of geophysical techniques to obtain subsurface information by analyzing different quality parameters. Application of geophysical methods, such as ground penetrating radar (GPR) high resolution electrical tomography and seismic refraction, when conducting reservoir or outcrop research, is a low aggression tool for the environment, allowing knowledge of the complete characteristics of the rock before performing more aggressive work, such as exploratory drilling which complements the investigations.

Currently, very advanced geophysical prospecting techniques are being applied in the CTM with excellent results with the most accurate techniques being GPS and 3D scanners, which allow the acquisition of accurate results, and advance the knowledge and delimitation of the factors affecting the outcrops and quarries enabling resource optimization, both economically and environmentally.

This paper is intended to reflect the benefits of the application of geophysical prospecting in the field of ornamental rock, analyzing the different techniques that the CTM has been applying for several years in various operations and materials, to obtain the maximum information required.

One of the techniques used is GPR and Figure 1 shows a diagram of our GPR equipment as an example of how an object can be detected. The basic GPR operation for non-destructive terrain exploration is shown in Figure 2.

In Figure 3 the penetration problems of the signal can be observed, derived from the land heterogeneity. The antennas used in this kind of research are from $25 \mathrm{MHz}$ to $250 \mathrm{MHz}$, exceeding the height of the cross over bench and it is interesting to identify different structures at this scale of resolution. Figure 4 is a sample of measurements made using $25 \mathrm{MHz}$ antenna and $250 \mathrm{MHz}$. Another technique used is electric tomography, which is specifically designed to determine the actual distribution of ground resistivity within the area covered to a certain depth range along a profile measurement.

The electrical tomography method consists of introducing a direct current across the electrode surface with a particular separation depending on the measurement device used and the desired investigation depth. Figure 5 shows an example of measurements with electrical tomography and Figure 6 shows an example of an electrical tomography profile.

The method of seismic refraction used by the CTM is based on determining the time of the first arrivals of $P$ waves from a known point (seismic source) to a set of receivers (geophones) located along a profile separated at a certain distance.

Figure 7 shows the principles of the seismic refraction method and Figure 8 the measurement of a seismic profile. In the end we obtain a profile where we show the speed of propagation of $P$ waves that will allow us to differentiate between different materials and conditions found in differentiating degrees of weathering, massive areas, and so on.

\section{Application in various material holdings}

In this section, various application examples of the geophysical techniques described above in various materials that make up the sector of ornamental rock are summarized.

Firstly, results from the application of the techniques in limestone deposits (marble limestone), then their application in granite quarries and finally in a reservoir of calcarenites.

The limestone site used is located in the Sierra de la Puerta of Cehegín, where a well-known stone in the area, called Crema Marfil, is quarried. This project focuses on an area of tremendous structural complexity, where the quarry is located. In this study 15 electric tomography profiles have been made as well as 13 GPR profiles georeferenced using GPS to study the quarry with different antennas and to observe qualities of solid rock, geological structure, etc. Figure 9 shows the GPR profile with $25 \mathrm{MHz}$ antenna in limestone.

The tomography carried out resulted in the precise definition of the structure of the area. In Figures 10 and 11 the different layers in a perpendicular cut can be seen. The presence of a limestone layer below the current heap can be observed, as well as the presence of the layer blocks at the base of the mountain that has produced differential erosion.

Seismic refraction (Fig. 12) has been used in this area for the study of the exploitable formation wall in contact with the Oligocene, and thus assess the various thicknesses and geotechnical parameters from the different propagation speeds of the seismic waves.

The application of these technologies in granite has been made in the area of the Hesperian Massif, for the analysis of a quarry in a granite batholith of medium and fine grain. The general approach of this research is to gain a thorough understanding of the deposit at depth. 
Espín de Gea, A., et al., 2017. Los métodos geofísicos como herramienta de mejora para... Boletín Geológico y Minero, 128 (2): $363-378$

39 GPR profiles were performed with two antennas, $25 \mathrm{MHz}$ to reach the maximum depth and detect major discontinuities of "soil" and $250 \mathrm{MHz}$ to obtain more resolution and detect the presence of small-scale discontinuities. In the GPR profiles (Figs. 13 and 14) you can see the correlation between one of the profiles of $25 \mathrm{MHz}$ and $250 \mathrm{MHz}$ with the survey.

The scan was performed to delineate the presence in the outcrop aplite dike (Fig. 15).

Other materials, on which significant research has been carried out by the Department of Geology, in the area of calcarenites of Hellin, have been defined from the geological point of view as coastal deposits with abundant presence of sedimentary structures. Figure 16 shows the scan profile made perpendicular to the structure. In figure 17 an image of the quarry is shown.

Different scan profiles that mark the existing structure in this outcrop have been obtained, where we find conglomerates in the floor and roof of the exploitable calcarenites.

\section{Results and discussion}

We found that the combination of various geophysical techniques in the investigation of deposits of ornamental rock provides high quality final results, since each of them separately presents different challenges and may cause some doubt in the interpretation, whilst their joint use allows us to complete all the technical data, minimizing the areas where problems can occur or fail separately.

This project shows that the geophysical techniques that have been carried out in these projects are an innovative tool to obtain accurate and truthful information about reservoir structure, allowing interpretation and cubage, essential for future work in the exploitations.

The difficulties of implementation are also important, for example GPR may have many problems with the presence of layers of thin material, something very common in our study area, although it is a technique which is easily applicable and very versatile in the field and which allows easy handling for ornamental rock extraction sites. Tomography is a difficult technique for ornamental rock quarries, due to the length of the profiles needed to reach our research depths, nevertheless it is very decisive and effective with materials with strong contrast between resistivity. Seismic investigation gives us valuable information on the state of deterioration of materials of the site which can be translated into a classification of grades of material, but one difficulty is the presence of many machines in the quarry area resulting in the appearance of noise in the measurements.

As you can see its application is not limited by the type of material as it has been applied in these examples with very satisfactory results.

\section{Introducción}

Bajo la denominación de piedra natural o roca ornamental se engloban aquellas rocas que, una vez extraídas y tras un proceso de elaboración, son aptas para ser utilizadas como materiales nobles de construcción, elementos de ornamentación, arte funerario o escultórico y objetos artísticos variados, conservando íntegramente su composición, textura y propiedades físico-químicas.

Ante la situación del sector a nivel mundial y la amenaza directa de los países emergentes, el sector de la piedra natural debe apostar por la calidad.

El sector debe plantearse el futuro desde la investigación, desarrollo e innovación tecnológica en los procesos y productos, como única vía de supervivencia en un mercado cada vez más competitivo.

La Asociación Empresarial de Investigación Centro Tecnológico del Mármol y la Piedra (CTM) desarrolla su actividad desde 1996 en el ámbito de la I+D+l y los servicios tecnológicos relacionados con la piedra natural, la formación técnica y el asesoramiento a las empresas del sector, siendo un centro puntero a nivel nacional en su especialidad y contando con una amplia experiencia en el conocimiento de los distintos tipos de yacimientos explotados como roca ornamental y de las rocas como materiales de construcción.

El proceso de extracción de la piedra natural tiene un alto impacto en el medio ambiente y está sujeto a una legislación cada vez más estricta y exigente, por lo que urge la necesidad de desarrollar tecnologías capaces de permitir un conocimiento de los yacimientos actualmente en explotación y de las zonas explotables futuras, que permitan una optimización de los recursos tanto económicos como de la afección al medio natural que nos rodea. Todo esto unido a la gran sensibilización que existe en nuestra sociedad para la protección de nuestro entorno natural, hace necesaria la utilización de tecnologías no invasivas, como las geofísicas, que sean capaces de obtener la máxima información posible, con un coste con- 
tenido y una aportación fundamental en la toma de decisiones por parte de la empresa a la hora de realizar la inversión.

Actualmente en el CTM se están aplicando técnicas de prospección geofísica muy avanzadas con gran apoyo en los sistemas topográficos más precisos como son GPS y Escáner 3D, que están permitiendo obtener unos resultados fiables y muy ajustados a la realidad comercial del yacimiento, conocedores de que el conocimiento y delimitación de los factores que afectan a los afloramientos y canteras, permiten una optimización del recurso tanto desde el punto de vista económico como medioambiental.

\section{Objetivos}

En este trabajo se pretende reflejar los beneficios que aporta la aplicación de la prospección geofísica al sector de la roca ornamental, analizando las diferentes técnicas que el CTM está desarrollando desde hace varios años en diferentes explotaciones y materiales, para obtener el máximo de información, necesaria para la toma de decisiones en la apertura de nuevas explotaciones o en la continuidad de las existentes.

Se analiza la aplicación de técnicas geofísicas con tres ejemplos en materiales muy representativos del sector donde se destacan los pros y los contras de cada una.

Para finalizar se exponen las conclusiones principales a las que tras el análisis de los resultados obtenidos se ha llegado.

\section{Técnicas aplicadas: georadar}

El georadar es un método de prospección electromagnética para el estudio no destructivo del subsuelo. La señal recibida se amplifica, digitaliza y almacena para posteriormente, mediante un adecuado procesado podamos adquirir información de las características, contenido y posibles anomalías del subsuelo. Entre la posible información obtenida de un georadar está la profundidad, orientación, tamaño y forma de objetos enterrados.

Se debe tener en cuenta que la única información fiable de la que disponemos es el tiempo de viaje de la señal electromagnética: tiempo que transcurre desde que la señal es emitida por la antena emisora, penetra en el terreno, encuentra una anomalía y se refleja el eco, captándose el evento en la antena receptora. A partir de estos datos y mediante un buen procesado se puede obtener una estimación fiable de la geometría del reflector.

En este trabajo se propone la utilización de esta técnica geofísica para la detección en función de la resolución requerida, de las diferentes discontinuidades, karstificación, materiales, etc., presentes en el yacimiento, con diferentes escalas y antenas, que nos va a permitir traducir en posibilidades de explotación de estos recursos como roca ornamental. Se evalúa el estado de fracturación de los macizos rocosos donde se ubican canteras de roca ornamental o afloramientos, detectando en zonas kársticas, cuevas, sifones, dolinas y demás elementos presentes en este tipo de terrenos y buscando las zonas masivas de máxima calidad.

La figura 1 muestra el esquema de nuestro georadar de impulso, en el que se ha dibujado, como ejemplo de posible objeto a detectar (denominado "blanco"), una tubería. El término "clutter" se refiere a posibles objetos que, por definición, no interesa detectar pero que producen señales reflejadas no deseadas que son también recibidas por la antena receptora. Obviamente la existencia de este tipo de objetos puede complicar enormemente la interpretación de los datos recibidos e incluso, en algunos casos, ocultar por completo a los blancos u objetos de interés haciendo imposible la detección de estos, para los estudios de roca ornamental estos datos son importantes debido a que nos aportan información del estado del material. Una manera de disminuir la incertidumbre de las medidas 2D viene de la mano de la incorporación de los georadares 3D que montan varias antenas en paralelo con amplio rango de frecuencias con el objetivo de realizar una malla tridimensional de perfiles equidistantes para luego realizar un modelado tridimensional de la zona de estudio.

Como podemos comprobar el sistema está compuesto por un generador de pulsos que excita la antena emisora, una antena receptora con sus elementos de adaptación de la señal, y finalmente una pantalla para visualizar en tiempo real el resultado obtenido.

En el ejemplo recibiremos una primera reflexión en tiempo cero debido a la interfaz entre la antena y el suelo, una reflexión debida a la interfase entre las capas 1 y 2 (siempre que tengan propiedades electromagnéticas distintas), una tercera reflexión debida a objetos "clutter" y finalmente una reflexión debida a la tubería que es nuestro objetivo.

En este tipo de sistemas la resolución y la profundidad máxima de penetración están relacionadas con la frecuencia de la antena: antenas de mayor frecuencia proporcionan mayor resolución pero menor profundidad, al contrario que las antenas de menor frecuencia que nos permiten penetrar más pero su resolución es 


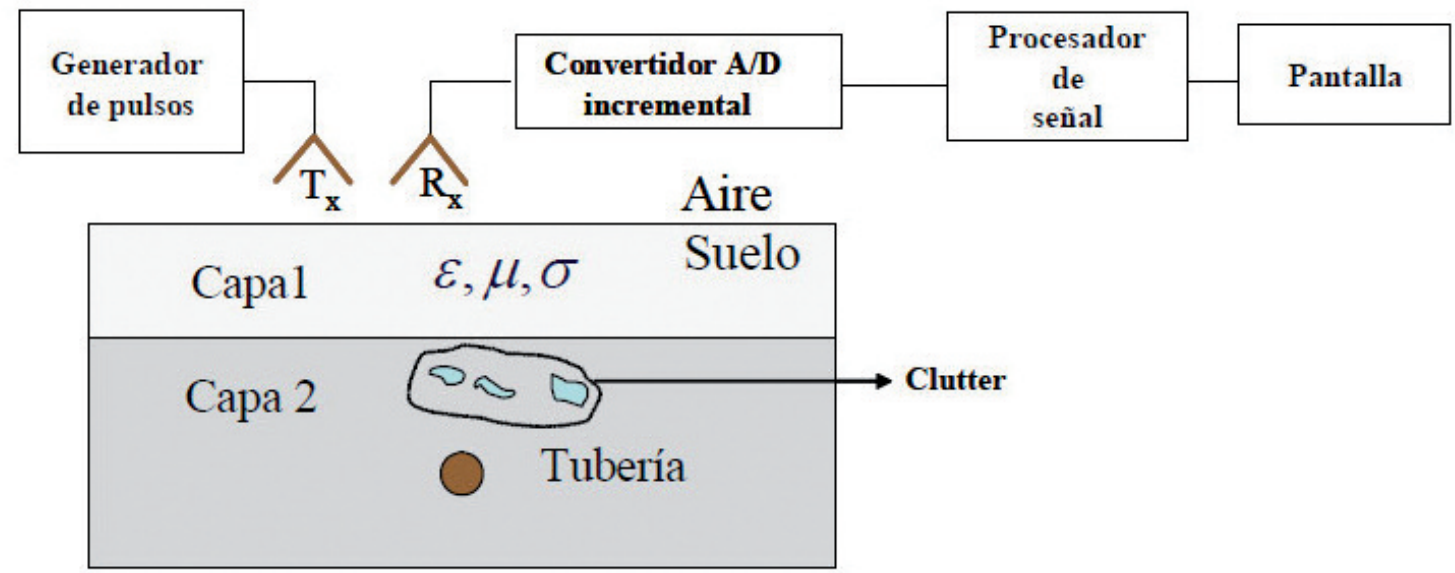

Figura 1. Ejemplo básico de un georadar para la exploración no destructiva del terreno (Gómez López, R.). Figure 1. Example of a basic GPR for non-destructive terrain exploration (Gómez López, R.).

menor. Consecuentemente, a la hora de elegir la frecuencia de la antena se debe mantener un compromiso entre profundidad y resolución. Aun así, la profundidad máxima de penetración no sólo depende de la frecuencia de la antena, se encuentra altamente influenciada por las características del terreno.

Para alta penetración $(10-40 \mathrm{~m})$ se utilizan antenas de 25-250 MHz con una resolución de 1-0.1 m; para estudios de los primeros 3-5 $\mathrm{m}$ del subsuelo se emplean antenas de $500 \mathrm{MHz}$, que proporcionan un buen compromiso entre penetración y resolución $(5 \mathrm{~cm}$ aproximadamente); en estudios de alta resolución se emplean antenas de alta frecuencia $(800 \mathrm{MHz}-2.3 \mathrm{GHz})$ que difícilmente penetran más allá de $1 \mathrm{~m}$ pero permiten localizar elementos con precisión centimétrica.

Como se ha podido comprobar el georadar tiene muchas ventajas, entre las que destacamos que forma parte de las llamadas técnicas no destructivas, que permiten obtener información del terreno sin perforarlo ni modificarlo, además son sistemas relativamente sencillos de aplicar con los que se puede obtener gran información y una profundidad de penetración bastante elevada.

En la imagen de la figura 2 se puede observar un claro ejemplo de aplicación efectiva de esta técnica con un radargrama realizado en una cantera donde se pueden apreciar las distintas discontinuidades, junto con zonas de alta calidad susceptibles de ser aprovechadas como roca ornamental. El objetivo de este perfil era determinar la profundidad, geometría y espaciamiento de las discontinuidades para planificar el diseño del avance de la cantera en función de las masas más potentes de material inalterado y también las posibilidades para su explotación.

No obstante, también tienen desventajas, entre las que destacamos que su buen o mal resultado depende principalmente de las características del terreno. Los terrenos homogéneos serán adecuados mientras que aquellos terrenos muy heterogéneos, porosos o con gran humedad reducirán el rendimiento del sistema. En zonas que no sean propicias para la utilización de este sistema, suele combinarse con otras técnicas geofísicas para arrojar luz a las posibles incertidumbres del georadar. Uno de los inconvenientes más importantes que se ha encontrado para su incorporación en los estudios de roca ornamental es la presencia de materiales finos como es el caso de arcillas, limos y margas que absorben la señal, lo que hace disminuir drásticamente la penetración (figura 3). Este tipo de materiales también se suelen encontrar en la superficie de contacto entre las antenas y el suelo cuando se realizan las medidas por los caminos con abundante circulación de vehículos. También la presencia de abundante vegetación provoca el mal contacto, levantando las antenas que provoca un mal contacto con la superficie del terreno.

El equipamiento del CTM está compuesto por una amplia gama de antenas apantalladas, que operan a unas frecuencias que van desde los $25 \mathrm{MHz}$ hasta los $2.3 \mathrm{GHz}$, con esto conseguimos adaptarnos a todas las situaciones posibles dentro de las necesidades del mundo de la roca ornamental, aunque en los trabajos de investigación minera las antenas más utilizadas van desde los $25 \mathrm{MHz}$ hasta los $250 \mathrm{MHz}$ (figura 4), sobre todo, son las que superan en profundidad la altura de más de un banco y una resolución interesante para identificar diferentes estructuras a esta escala. Desde el comienzo las medidas se corrigen mediante estación total y GPS centimétrico RTK, porque, obtener una detallada precisión en las medidas 


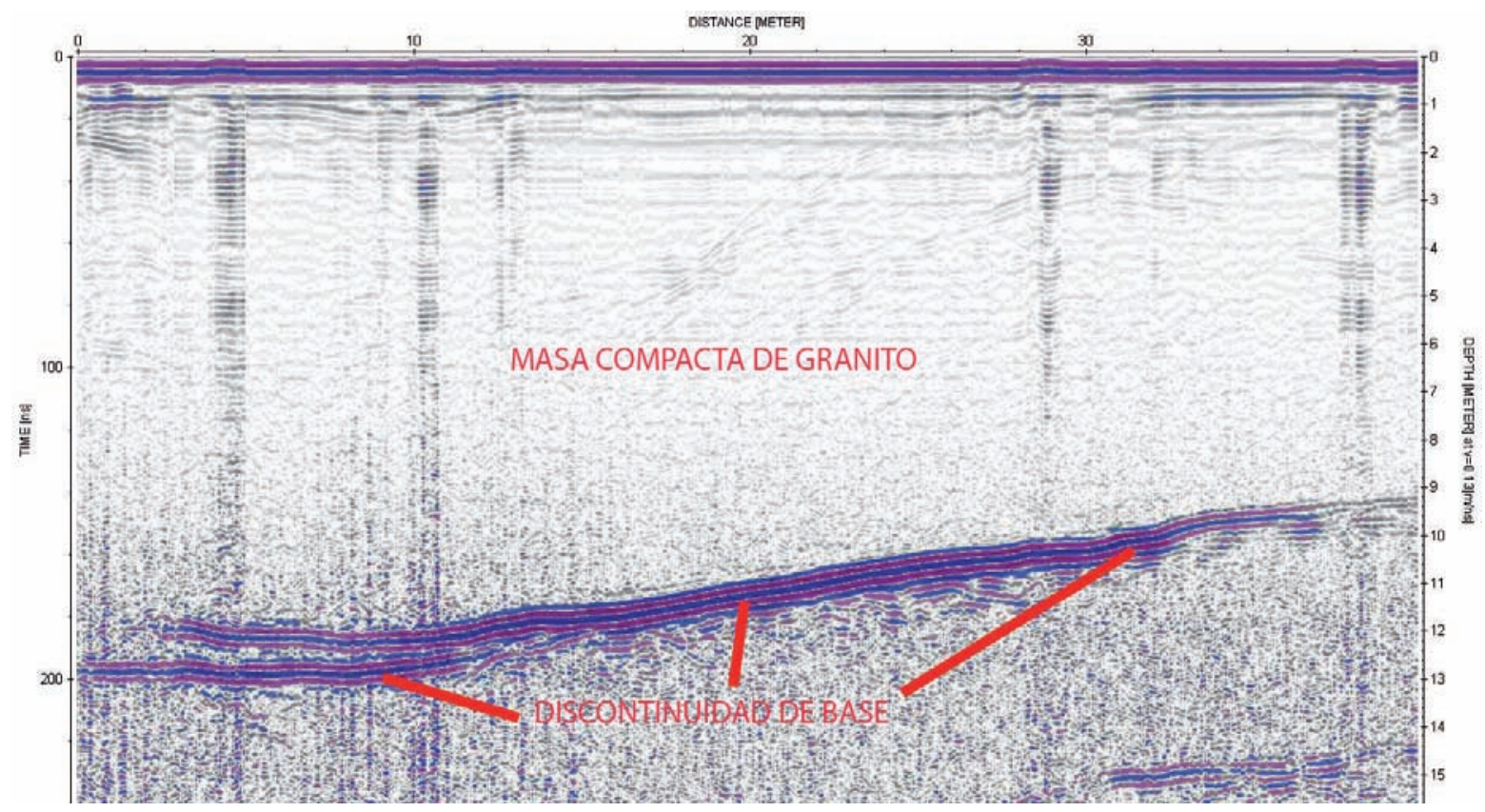

Figura. 2. Radargrama con gran penetración e identificación de la calidad del material y distribución de las discontinuidades. Figure 2. Radargram with high penetration and material quality identification and distribution of discontinuities.

se traduce en una interpretación ajustada a la realidad y a los objetivos perseguidos, por lo que la interpretación se facilita de manera importante. La topo- grafía nos permite obtener imágenes tridimensionales de la zona de estudio, lo que nos hace trabajar siempre de forma real y detallada.

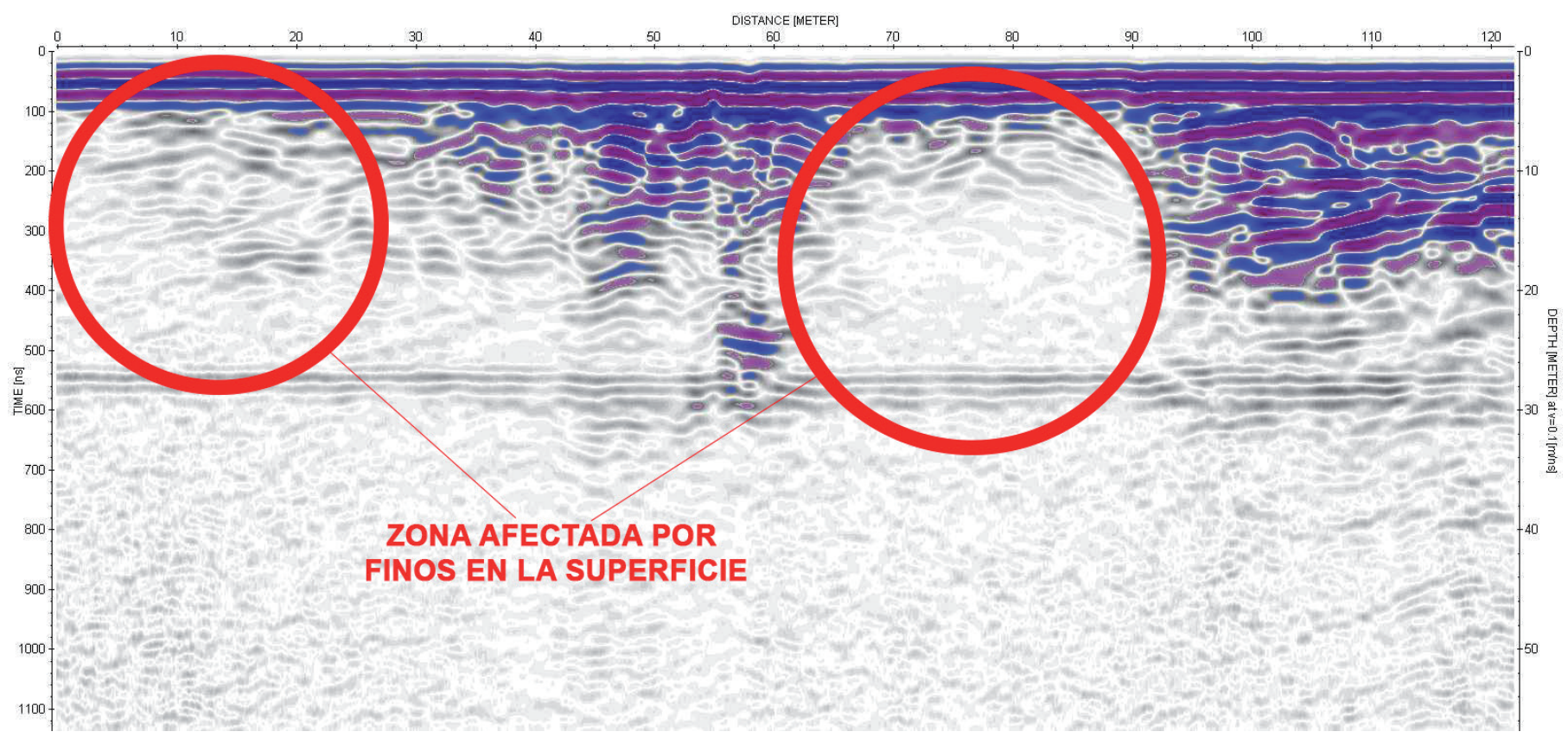

Figura 3. Problemas de penetración de la señal, derivados de la heterogeneidad del terreno y presencia de finos.

Figure 3. Penetration problems of the signal, derived from the land heterogeneity and fine materials. 

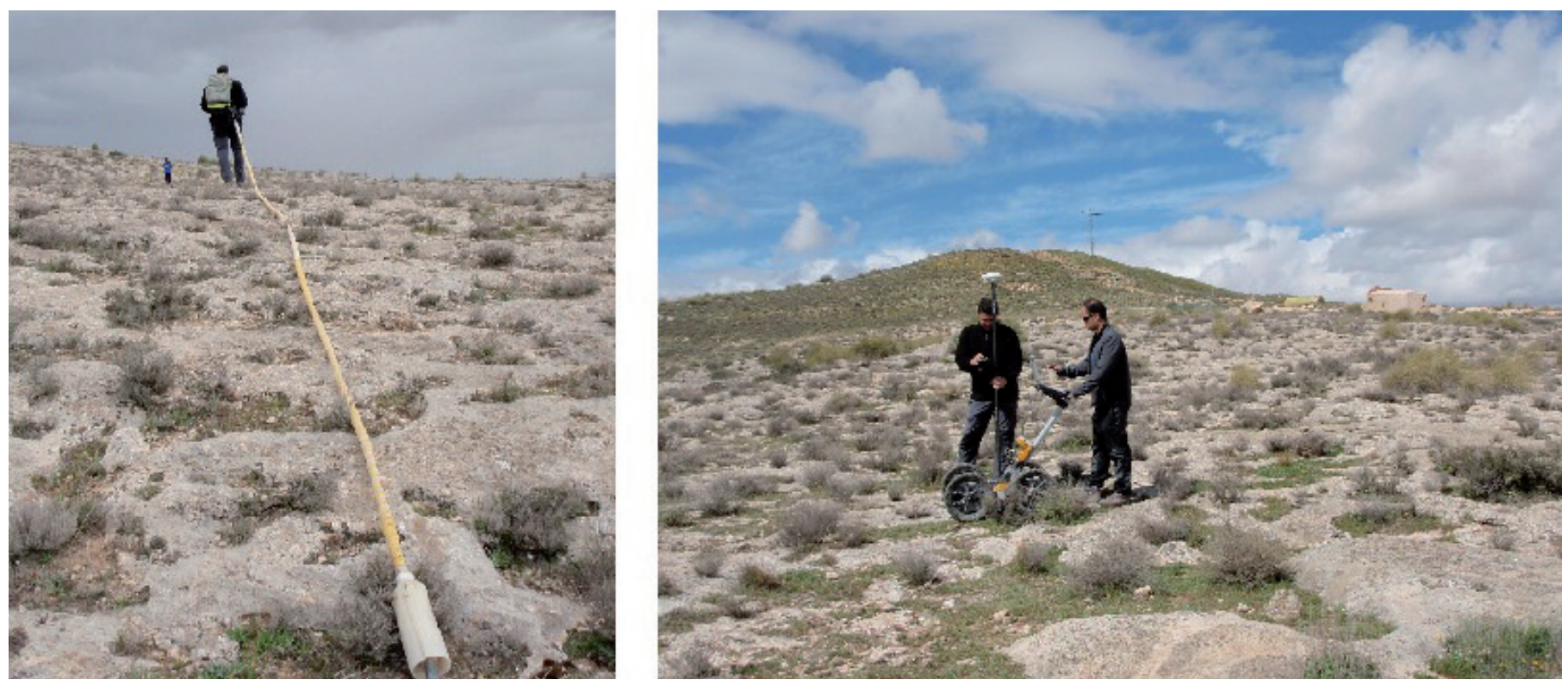

Figura 4. Realizando medidas de GPR con antena de $25 \mathrm{MHz}$ (foto de la izquierda) y antena de $250 \mathrm{MHz}$ (foto de la derecha).

Figure 4. Measurements of GPR using $25 \mathrm{MHz}$ antenna (pictured left) and $250 \mathrm{MHz}$ antenna (pictured right).

\section{Técnicas aplicadas: tomografía eléctrica}

En su sentido más amplio la tomografía eléctrica es una técnica geofísica geoeléctrica para el estudio del subsuelo que consiste en determinar la distribución de un parámetro físico característico del mismo, dentro de un ámbito espacial limitado, a partir de un número muy elevado de medidas realizadas desde la superficie del terreno o desde sondeos.

La tomografía eléctrica tiene por objetivo específico, determinar la distribución real de la resistividad del subsuelo en un ámbito comprendido, hasta un cierto rango de profundidad a lo largo de un perfil de medida, a partir de los valores de resistividad aparente obtenidos mediante medidas realizadas por métodos convencionales de corriente continua (Granda, A. y Cambero, J.C. 2012).

Los sistemas basados en tomografía eléctrica, se utilizan para la detección de zonas estructuralmente débiles, relacionadas con la baja resistencia eléctrica de estas zonas que para el sector de la roca ornamental se traduce en zonas de alteración o baja calidad que posiblemente no sean aptas para su explotación. Estas zonas suelen ser partes del macizo fracturadas en las que se introducen materiales finos, cuyo contenido en agua es mayor, o donde la composición del material cambia completamente. Cada tipo de material presenta un rango de resistividad "real" más o menos característico aunque puede variar según las condiciones de la zona de estudio.
Un factor clave de esta técnica es el número y distribución de las medidas de campo ya que de él depende tanto su resolución como la profundidad de investigación. Este método permite discriminar zonas de roca masiva, resistividades altas, frente a zonas con mayor contenido en finos o humedad por lo que tenemos resistividades bajas.

Se realizan perfiles con múltiples electrodos en línea (figura 5), o irregulares, que nos permiten identificar en profundidad los materiales y las distintas discontinuidades del yacimiento. La utilización de líneas paralelas de electrodos permite la obtención de imágenes en pseudo 3D de la resistencia del subsuelo o también la utilización de una malla de electrodos permite la creación del modelo tridimensional de resistividades del terreno. Uno de los problemas de esta técnica para utilizar en canteras de roca ornamental es la longitud necesaria para una profundidad de investigación adecuada y la presencia de muchos obstáculos que limitan la implantación de este tipo de dispositivos.

El resultado final de este tipo de estudio es una sección distancia-profundidad (figura 6) con la distribución de la resistividad real del subsuelo, esta sección tiene $355 \mathrm{~m}$ de longitud, 72 electrodos y espaciado $5 \mathrm{~m}$, en la que somos capaces de detectar distintas estructuras geológicas y parámetros físicos utilizados tanto en el ámbito de la geología como de la geotecnia. Las medidas de resistividad aparente del terreno para un estudio mediante tomografía 


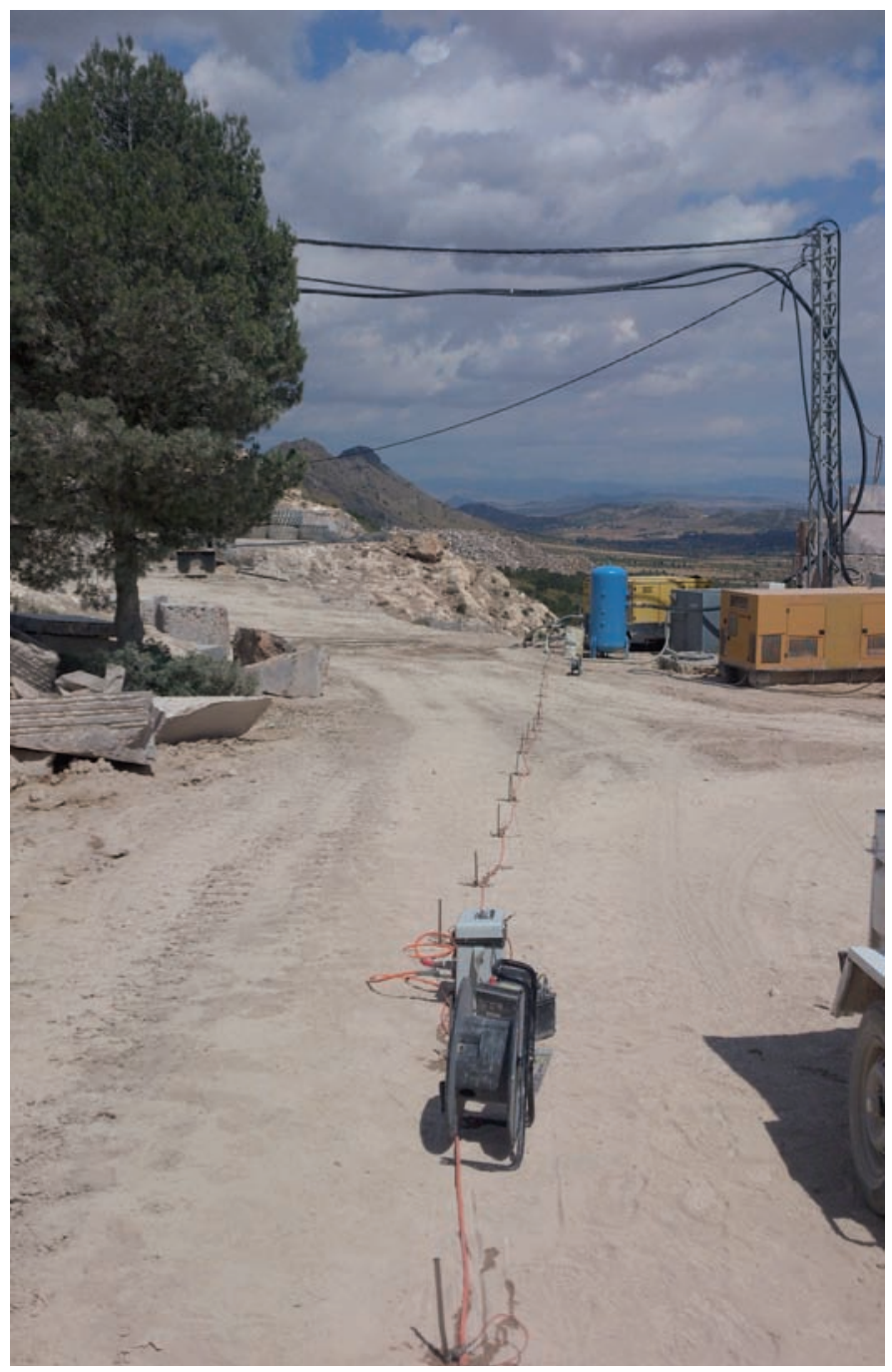

Figura 5. Toma de medidas con tomografía eléctrica.

Figure 5. Taking measurements with electrical tomography.

eléctrica se efectúan mediante técnicas de corriente continua con posibilidad de empleo de una gran variedad de dispositivos en lo que a la distribución de los electrodos se refiere. Entre los más habitualmente utilizados y que el CTM está utilizando, cabe citar los dispositivos polo-dipolo, dipolo-dipolo, Schlumberger, etc.

En el sector de la roca ornamental superar los 100 $\mathrm{m}$ de profundidad es poco frecuente debido a las características de los yacimientos que se pretenden estudiar pudiendo llegar esta técnica hasta a más profundidad, identificando discontinuidades, calidades de material que tengan variación de resistividad suficiente para ser interpretada.

Es fundamental la detección y caracterización de fallas determinando su zona de influencia, rumbo, buzamiento, extensión en profundidad, contactos entre unidades litológicas de diferente naturaleza, determinando la morfología y localización precisa de tales discontinuidades. También la detección y caracterización de accidentes kársticos, tales como cavidades, rellenos arcillosos, distintas escalas de fracturas, etc.

Las medidas realizadas con tomografía son georreferenciadas mediante estación total y estación total GPS con lo que nos aseguramos que la topografía no será una fuente de error, tanto en el procesado como en la interpretación.

Los estudios realizados sobre las rocas carbonatadas utilizadas como roca ornamental se ubican en la zona superficial de estas grandes masas y donde cobra mucha importancia la diferenciación del epikarst que es una zona alterada de los macizos carbonáticos situada inmediatamente bajo la superficie del terreno o bajo el suelo edáfico, cuando éste está presente (Williams, 2008), por debajo de este se encuentra el resto de la masa inalterada o alterada por las grandes diaclasas. Delimitar esta zona y el grado de meteorización es fundamental para nuestro trabajo porque la gran mayoría de las canteras se ubican en esta zona y cuantificar esta capa, que puede ser muy variable, es fundamental para la viabilidad de una explotación.

\section{Técnicas aplicadas: sísmica}

Los métodos sísmicos se basan en el estudio de la propagación de ondas elásticas a través de un medio de propiedades mecánicas determinadas. Cuando una onda elástica se propaga en un medio y encuentra una discontinuidad en las propiedades mecánicas del medio, sufre varios fenómenos físicos de relevancia, reflexión, refracción, transmisión y en algunos casos difracción.

El estudio del comportamiento de las ondas elásticas ante estos fenómenos permite estudiar las características elásticas del medio y extraer, de manera directa parámetros tan importantes como la velocidad de propagación, la atenuación, densidad, etc. Estos parámetros son de vital importancia para delimitar la estructura geomecánica del subsuelo y se emplean habitualmente en exploración geofísica en todos los ámbitos de aplicación desde estudios a pequeña escala con utilización de mazas o explosivos, hasta los estudios de estructura a escala planetaria mediante el empleo de ondas sísmicas generadas por grandes terremotos.

El método utilizado por el CTM es la sísmica de refracción, y se basa en determinar los tiempos de primera llegada de las ondas $\mathrm{P}$ desde un punto cono- 


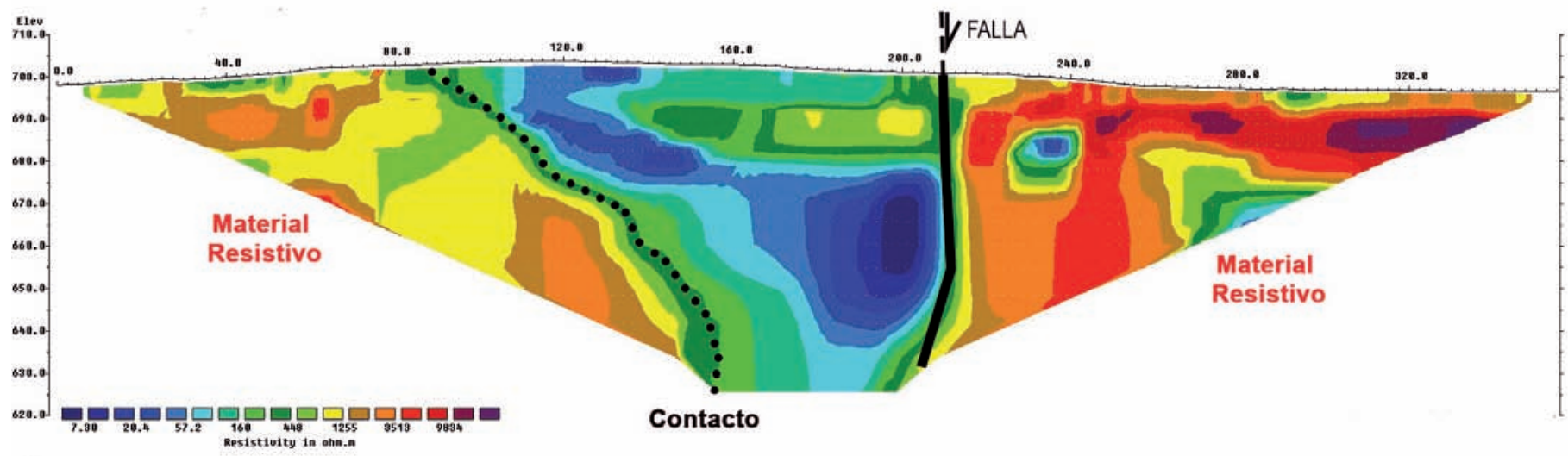

Figura 6. Perfil de tomografía eléctrica donde se pueden ver varias estructuras geológicas.

Figure 6. Profile of electrical tomography where you can see different geologic structures.

cido (fuente sísmica) hasta una serie de receptores (geófonos) situados a lo largo de un perfil donde se disponen éstos. Al conocer el tiempo de recorrido que las ondas $\mathrm{P}$ emplean en cubrir la distancia que separa la fuente y los geófonos, se puede determinar la velocidad de propagación del medio situado entre ambos. A partir de estos valores de velocidad se puede adquirir un modelo de calidades del subsuelo donde podemos tener capas de velocidad constante y espesor variable. Este método se utiliza cuando se quiere realizar un modelo de calidades de la zona de estudio o contrastar otros resultados obtenidos con otras técnicas, siendo este un método eficaz y relativamente rápido con menor limitación a la hora de implantar los perfiles por su menor longitud.

Al final obtenemos un perfil donde tenemos representada la velocidad de propagación de las ondas $P$ que nos va a permitir diferenciar los distintos materiales y las condiciones en las que se encuentran, diferenciando grados de meteorización, zonas masivas, etc.

Como hemos mencionado anteriormente este método usa las ondas de primera llegada $\mathrm{P}$, pero tam-

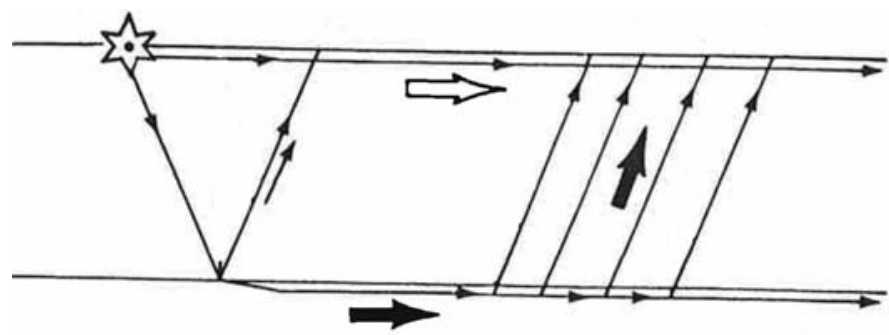

Figura 7. Principios del método de sísmica de refracción (Sharma, P.V. 2004).

Figure 7. Principles of the seismic refraction method (Sharma, P.V. 2004). bién se pueden adquirir las ondas $\mathrm{S}$ variando el tipo de geófonos, por lo que una vez conocemos estas dos velocidades de una zona, se pueden extraer de las medidas los módulos elásticos que pueden ser interesantes para proyectos variados dentro de la vida de la explotación.

\section{Aplicación en varias explotaciones y materiales}

En este apartado se recogerán distintos ejemplos de aplicación de las técnicas geofísicas anteriormente descritas en varios materiales que conforman el sector de la roca ornamental.

En primer lugar se exponen los resultados obtenidos con la aplicación a yacimientos de calizas (calizas marmóreas), después continuaremos con su aplicación en canteras de granito y para finalizar, en un yacimiento de calcarenitas.

El yacimiento de calizas analizado está ubicado en la Sierra de la Puerta de Cehegín donde se extrae un material muy conocido en el sector, llamado Crema Marfil Sierra de la Puerta. Este proyecto se centró en una zona de tremenda complejidad estructural, donde está ubicada la cantera.

La investigación realizada ha constatado la existencia de numerosas fallas que trastocan de manera fehaciente e importante la geometría de la capa explotada, llegando incluso a producirse repetición de capa dentro de la propia cantera, en una zona compleja de por si, por la existencia de un franco invertido.

Se trata de una serie monoclinal con un buzamiento acusado hacia el SE. Existen dos miembros competentes que resaltan entre miembros de naturaleza más blanda. Los miembros competentes son las calizas color crema de edad Eoceno superior (explotables) y las calizas tableadas del Oligoceno. 


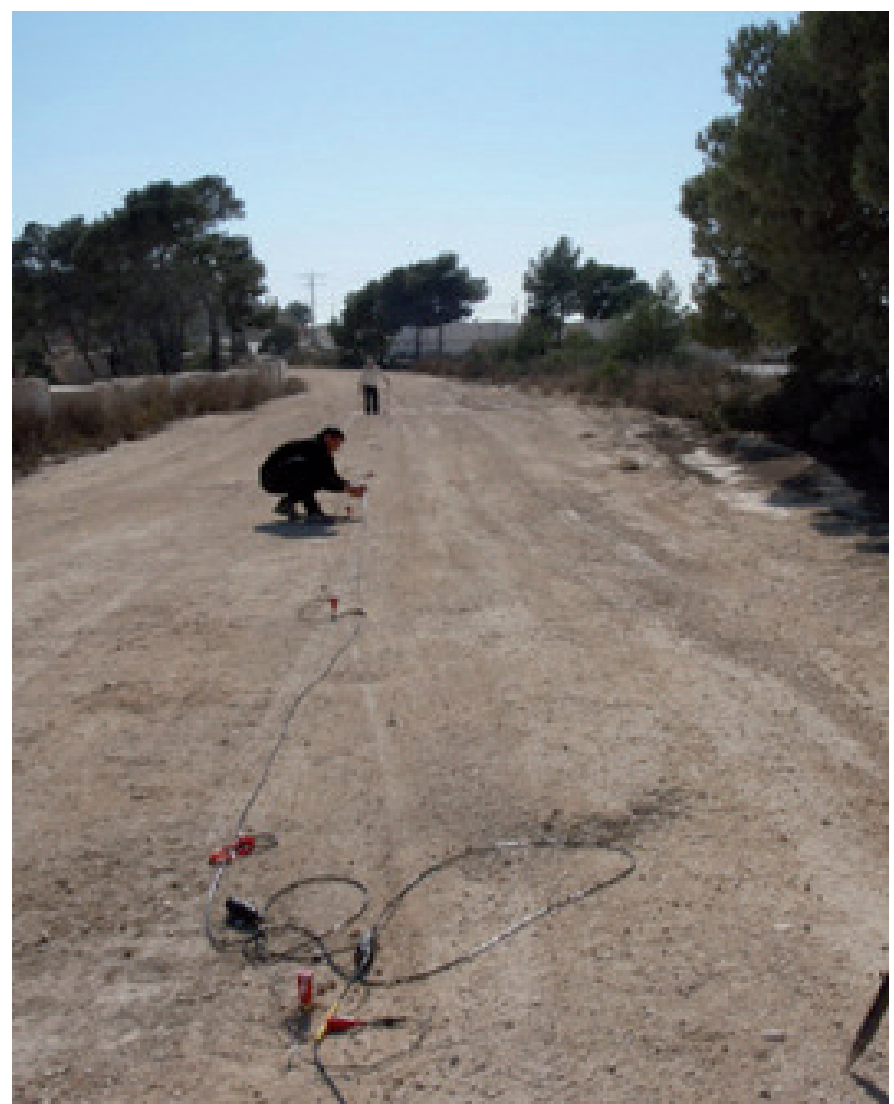

Figura 8. Toma de medidas de sísmica de refracción.

Figure 8. Seismic refraction measument.

El Eoceno superior lo forman las calizas color crema, masivas con algas, numerosos restos de equínidos y otros microfósiles como discocyclinas, actinocyclinas, heterostegina, etc. Este es el miembro explotable en la Sierra de la Puerta. AI SO aparecen las margas del Eoceno medio y al NE las margas miocenas, siendo ambos los miembros incompetentes.

Hacia el NE entramos dentro de los dominios del "Sinclinal de Moratalla", cuyo núcleo está formado por margas miocenas. Hacia el SO, a partir de las margas del Eoceno medio encontramos numerosos niveles, que pertenecen tanto al Paleógeno como al Cretácico, hasta alcanzar el Trías Subbético.

Este proyecto se planteó por el desconocimiento del yacimiento existente debido a la presencia de una gran capa de bloques en la base de la sierra y la aparición de varias fallas con salto bastante amplio que desplazaban los bloques, haciendo desaparecer la capa de calizas en su estado actual. Nuestro objetivo era cartografiar los contactos actuales en superficie y averiguar la estructura en profundidad de la capa explotable, y si debajo de esos bloques existía capa.
Este estudio ha consistido en la realización de 15 perfiles de tomografía eléctrica y 13 perfiles de georadar georreferenciados mediante GPS para estudiar la cantera con diferentes antenas y observar calidades del macizo de roca, estructura geológica, huecos y la dirección de las fracturas más importantes. En la figura 9 se presenta un ejemplo del trabajo realizado donde se puede observar, de derecha a izquierda del perfil cómo se distribuye la caliza en profundidad por debajo de la zona de bloques, apreciándose claramente su distribución y buzamiento, junto con la presencia de una gran discontinuidad con buzamiento hacia el comienzo del perfil. Este perfil se ha realizado con la antena de $25 \mathrm{MHz}$ y llega a profundizar hasta $40 \mathrm{~m}$. Una vez analizado este perfil junto con otros, nos permitió identificar la presencia a los 330 $\mathrm{m}$ de una falla que produce la desaparición de la caliza explotable y lo que muestra el perfil entre los 330 y $200 \mathrm{~m}$ es la presencia de bloques de forma heterogénea. Se pueden apreciar reflexiones múltiples que han sido eliminadas.

La tomografía realizada ha facilitado la definición precisa de la estructura de la zona con la identificación de las diferentes capas hasta el muro, zona de bloques y la geometría de la capa en profundidad. En la figura 10 y 11 se ilustran los perfiles de tomografía de $355 \mathrm{~m}$ de longitud, 72 electrodos y espaciado $5 \mathrm{~m}$, en los que se diferencian las distintas capas en un corte perpendicular, donde se puede apreciar la presencia de la capa de calizas por debajo de la escombrera actual al igual que también la capa de bloques en la base de la sierra que ha producido una erosión diferencial. Con el conjunto de la investigación realizada se han obtenido los objetivos perseguidos al comienzo del proyecto que eran detectar la estructura geológica de la zona y la geometría de las capas por debajo de la escombrera y la definición de la zona de bloques, también se ha constatado que la existencia de la capa de bloques en la base de la sierra es consecuencia de un desplome en la zona alta de la misma. También se ha podido constatar, tanto en el GPR como en la tomografía eléctrica la calidad de los materiales presentes en esta zona.

La sísmica de refracción se ha utilizado en esta zona para el estudio de la zona de bloques para detectar los bolos explotables de las cuevas existentes dentro de la formación explotable en contacto con el Oligoceno y así evaluar la presencia del material, calidad, presencia de finos, cavidades y parámetros geotécnicos a partir de las diferentes velocidades de propagación de las ondas sísmicas.

La aplicación de estas tecnologías en el granito se ha realizado en el ámbito del Macizo Hespérico, para el análisis de varias canteras en un batolito de grani- 


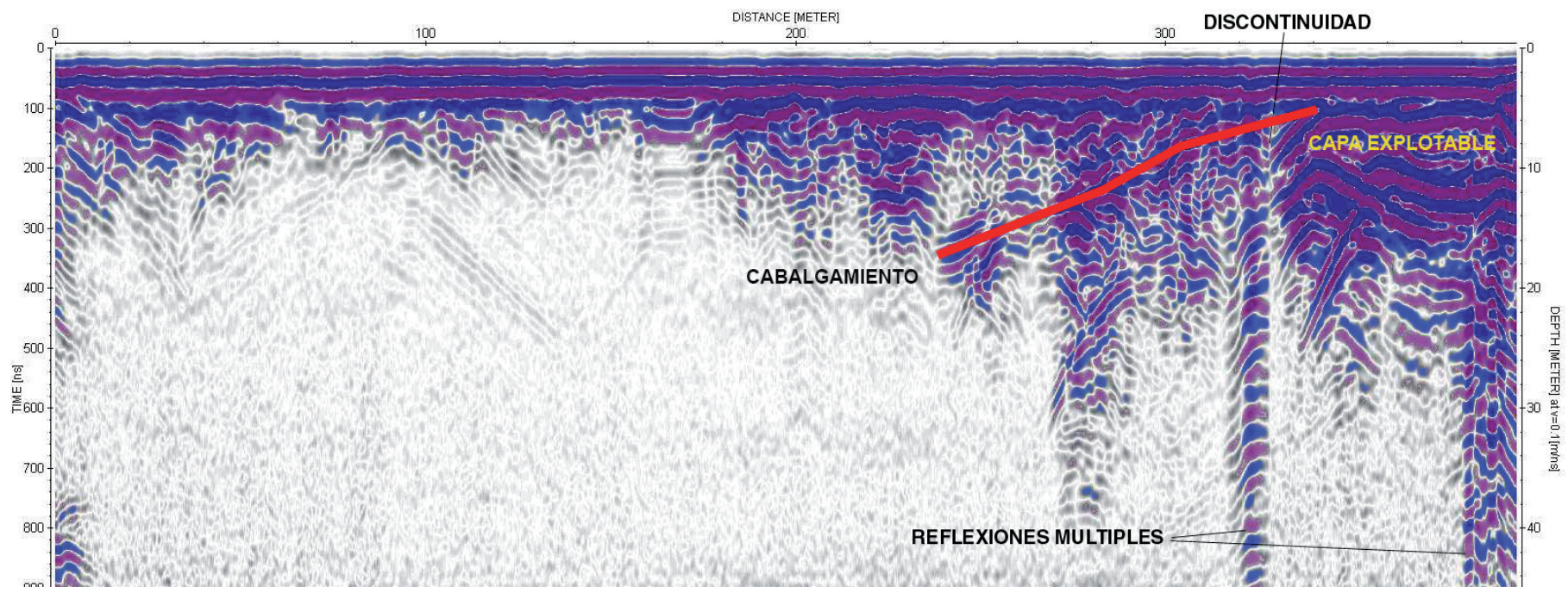

Figura 9. Perfil de georadar con antena de $25 \mathrm{MHz}$ en calizas.

Figure 9. GPR profile with $25 \mathrm{MHz}$ antenna in limestone.

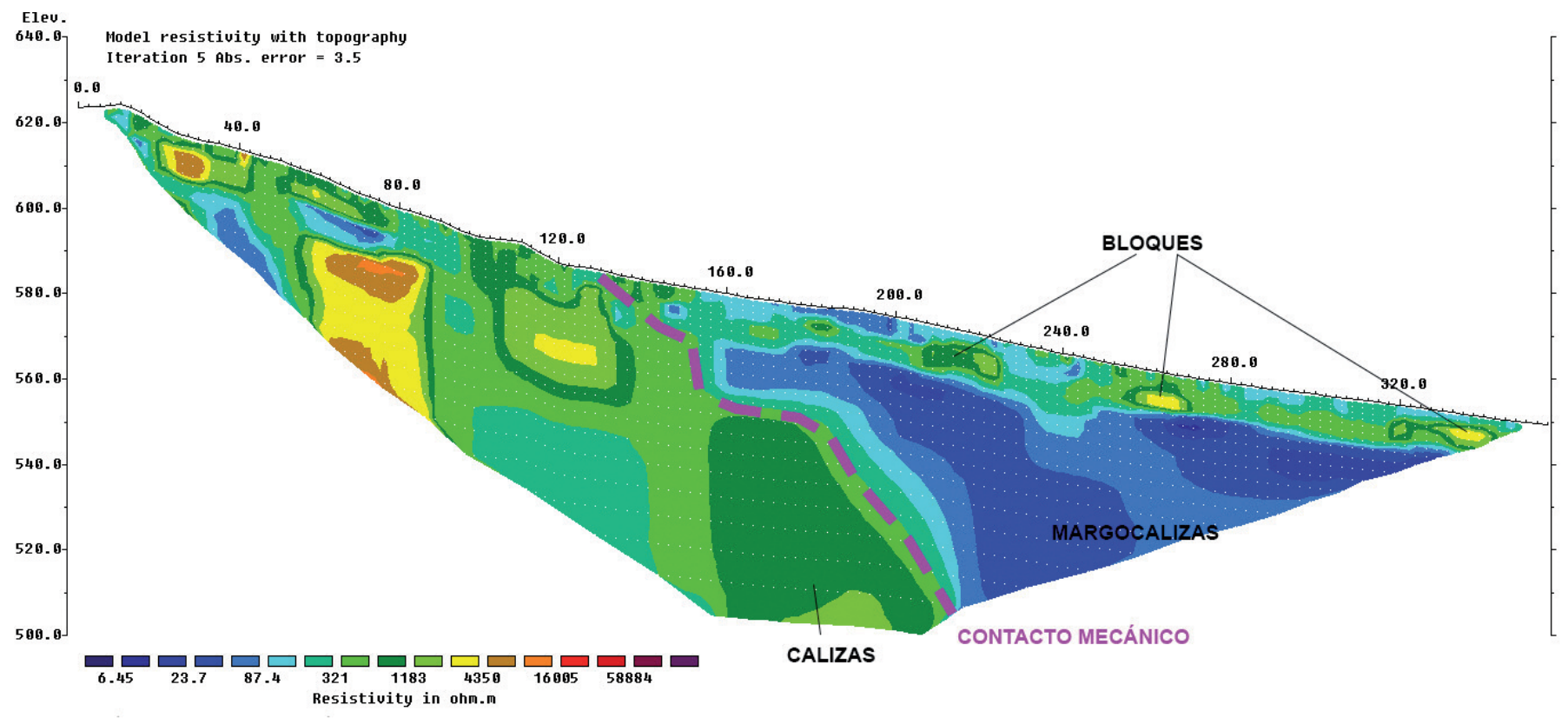

Figura 10. Perfil de tomografía con la zona de bloques.

Figure 10. Tomography profile with the area of blocks.

to de grano medio y fino que se integra dentro de los denominados granitos de dos micas y están siempre asociados a los granitos con megacristales a los cuales pasan gradualmente. El planteamiento general de esta investigación, es obtener un conocimiento exhaustivo del yacimiento en profundidad, los distintos tipos de granito, potencia del granito aprovechable, geometría de las discontinuidades con respecto a las masas de granito que ocupan todo el yacimiento, las zonas donde estas discontinuidades se presentan de manera intensa y como final, localizar las masas de granito con más potencia, sin presencia de discontinuidades.

Se realizaron 39 perfiles de georadar con dos antenas, de $25 \mathrm{MHz}$ para llegar a la máxima profundidad y detectar las grandes discontinuidades de "suelo", y con $250 \mathrm{MHz}$ para obtener más resolución y obtener la presencia de las discontinuidades de pequeña 


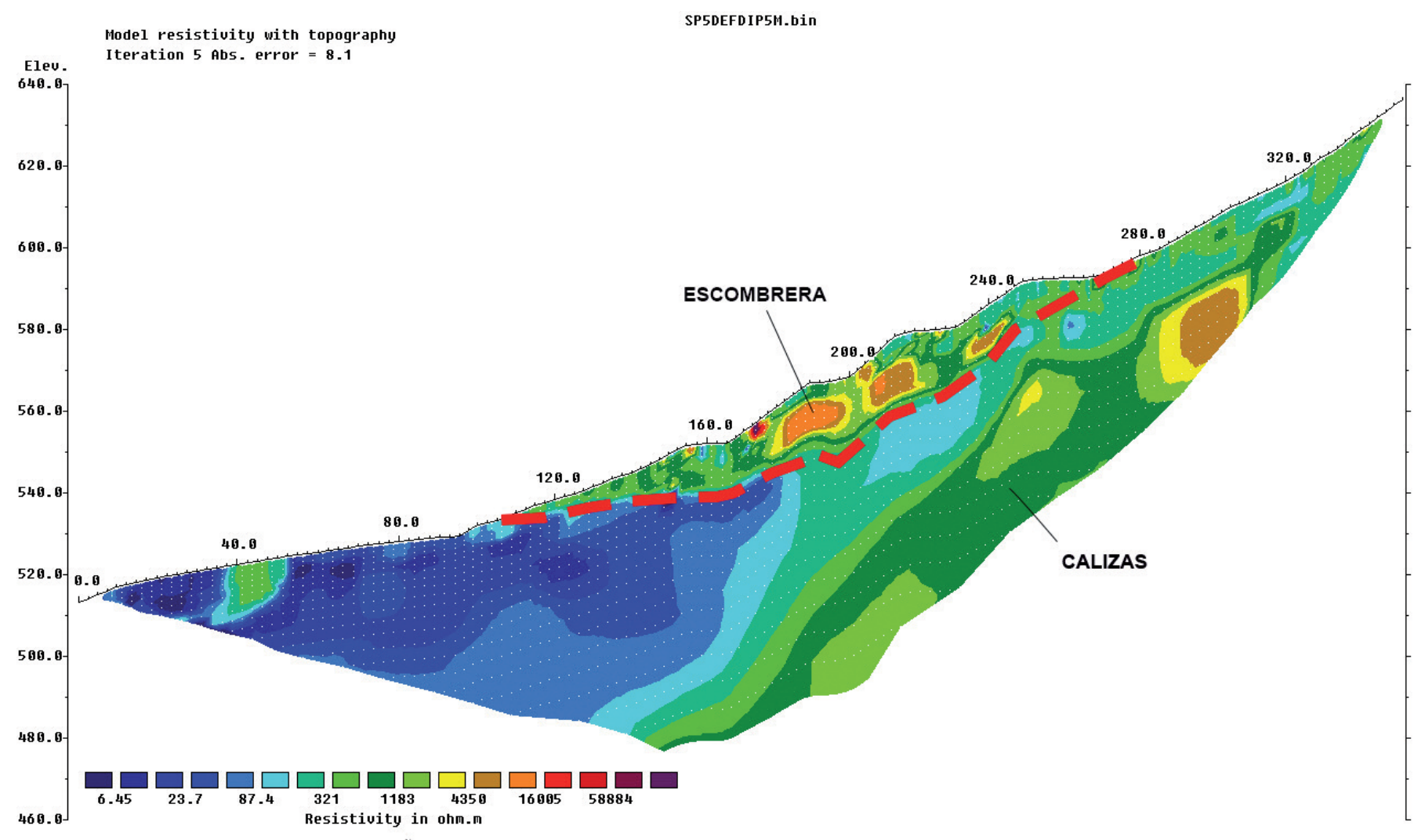

Figura 11. Perfil de tomografía con la escombrera cubriendo el material explotable.

Figure 11. Tomography profile where the dump is covering the exploitable material.

escala. Este perfil indica claramente el buzamiento de las discontinuidades de muro en esta zona con un espesor de la $1^{\text {a }}$ capa de unos $3 \mathrm{~m}$ y luego otra hasta los $7 \mathrm{~m}$ de profundidad. Se acuñan las discontinuida-

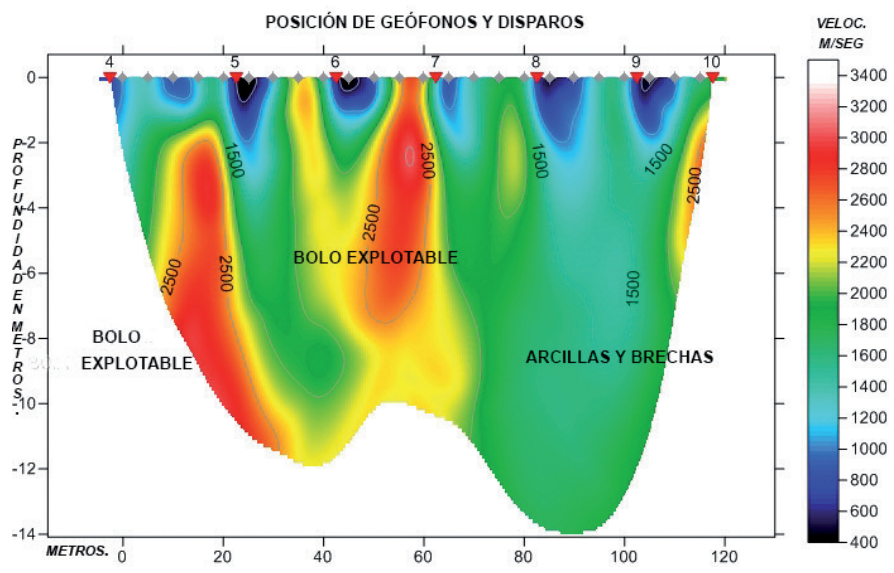

Figura 12. Perfil de sísmica de refracción con identificación en profundidad de la zona de bolos de caliza explotable.

Figure 12. Seismic refraction profile with identification in depth of the exploitable limestone boulder zone. des de muro hacia el final del perfil. Sigue habiendo fracturación vertical fuerte, que puede ser interesante para dimensionar un frente hacia el final del perfil, donde aumenta el espesor de las masas de granito debido al cambio de buzamiento de las discontinuidades y sobre todo en el centro del perfil donde el granito es más compacto.

Tras los datos obtenidos y el análisis de los perfiles realizados, se pudo constatar claramente la existencia de unas discontinuidades verticales con o sin estrías de falla, debido a esfuerzos sufridos por la masa de granito, y discontinuidades por descompresión en forma de lonchas de escamación superficial muy importantes en la cantera, que son las que controlan la explotación.

Se han detectado zonas donde las condiciones del granito son muy favorables para una explotación con frentes altos y donde la presencia de discontinuidades muy marcadas es mínima y las potencias son muy elevadas.

Estas zonas han sido posteriormente sondeadas con recuperación de testigo continuo, analizadas con interés para roca ornamental y corroborar así la validez de los resultados adquiridos con el georadar, 
obteniéndose una correlación casi perfecta entre las masas compactas de granito reflejadas en estos perfiles y en las zonas delimitadas, con las discontinuidades encontradas en los sondeos, proporcionando una información valiosa para la empresa y que se presenta fundamental para la futura planificación de las labores mineras. En la figura 13 y 14 se puede apreciar las correlación entre uno de los perfiles de 25 $\mathrm{MHz}$ y $250 \mathrm{MHz}$ con el sondeo realizado.

El sondeo se encuentra a $32 \mathrm{~m}$ del inicio del perfil de $250 \mathrm{MHz}$ y del perfil con $25 \mathrm{MHz}$ se localiza a $41 \mathrm{~m}$. En la figura 13 se puede observar el sondeo (línea roja) con varias discontinuidades marcadas en verde que debido a la resolución no se distinguen con esta antena y donde se puede apreciar que no se han tocado con este sondeo discontinuidades de gran escala como la que se aprecia a unos 22 metros de profundidad, marcada con un circulo cian y que corresponde a una superficie irregular que se extiende por el perfil con grandes reflexiones y de gran importancia para una futura explotación. El perfil de $250 \mathrm{MHz}$ (figura 14), nos indica que las discontinuidades encontradas en el sondeo (líneas verdes) corresponden con unas reflexiones muy marcadas a esa profundidad en el perfil y posteriormente, el sondeo va profundizando no encontrándose ninguna más hasta el final del sondeo, como se puede ver en el perfil donde no se aprecia ninguna reflexión, aunque cabe destacar que a partir de los $11 \mathrm{~m}$ aumenta el ruido, y pese a esto, no hay reflexiones en esa zona del perfil, ni en el testigo extraído existen discontinuidades.

La tomografía se ha realizado para delimitar la presencia en el afloramiento de un dique de aplitas.

En el perfil se observa una zona más resistiva en la parte superficial, con una potencia de unos cinco metros y presencia de bolos y discontinuidades. A partir de esta profundidad se puede observar una franja menos resistiva donde el granito puede estar alterado.

Esta capa tiene una potencia aproximada de $10 \mathrm{~m}$ buzante hacia el final del perfil que termina en un contacto con dos grandes masas de granito compacto separadas por una franja vertical que corresponde con la continuación del dique de aplitas con unos 10 $\mathrm{m}$ de potencia. Pasando esta zona, se encuentra en esta profundidad otra masa de granito compacto.

Otro de los materiales donde se han realizado, por parte del Departamento de Geología, investigaciones importantes, han sido en varias canteras de calcarenitas en la zona de Hellín, provincia de Albacete, defi-

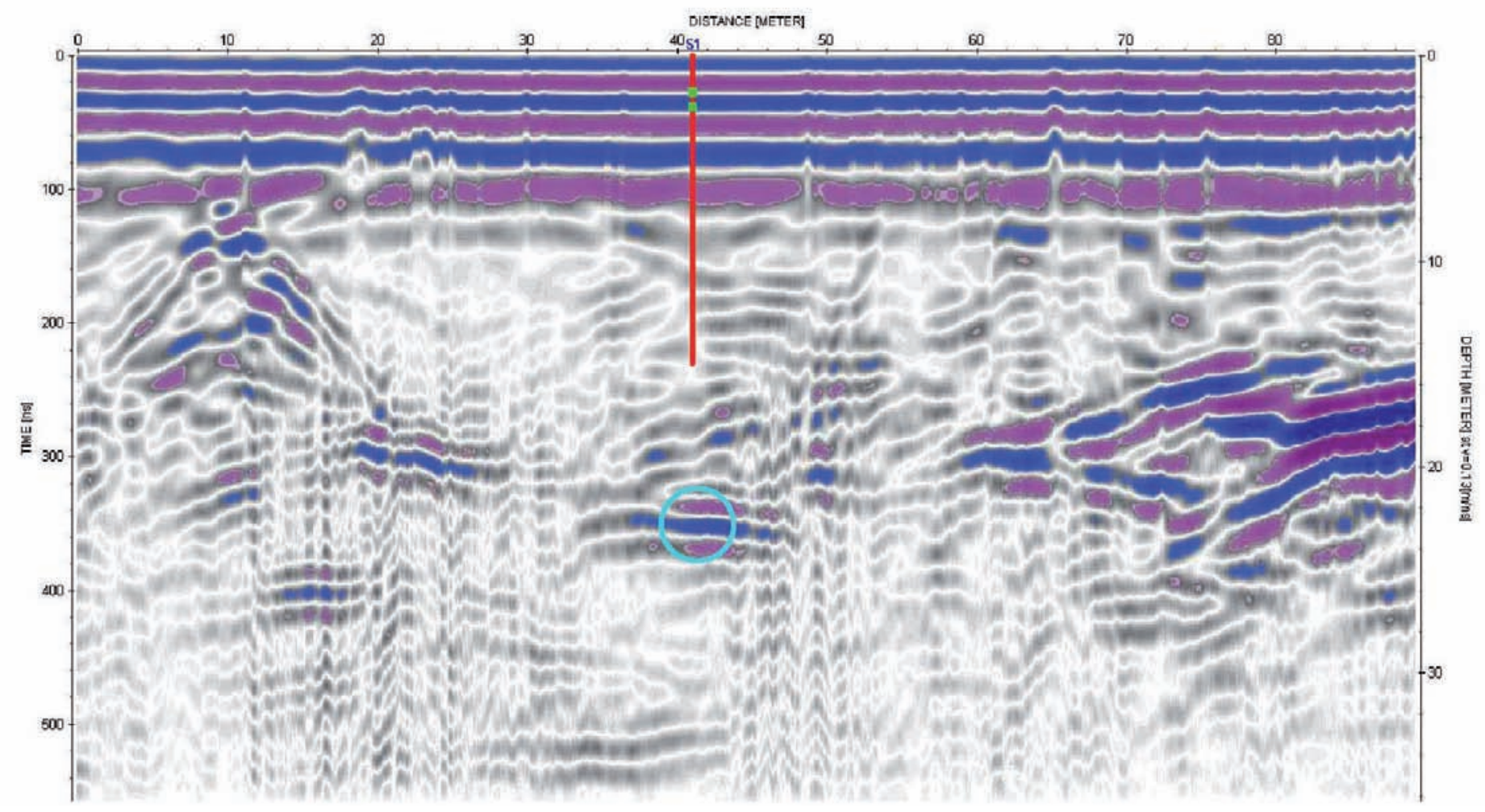

Figura 13. Perfil de georadar con antena de $25 \mathrm{MHz}$ en granito junto al sondeo estudiado.

Figure 13. GPR profile with $25 \mathrm{MHz}$ antenna in granite and studied.drill. 


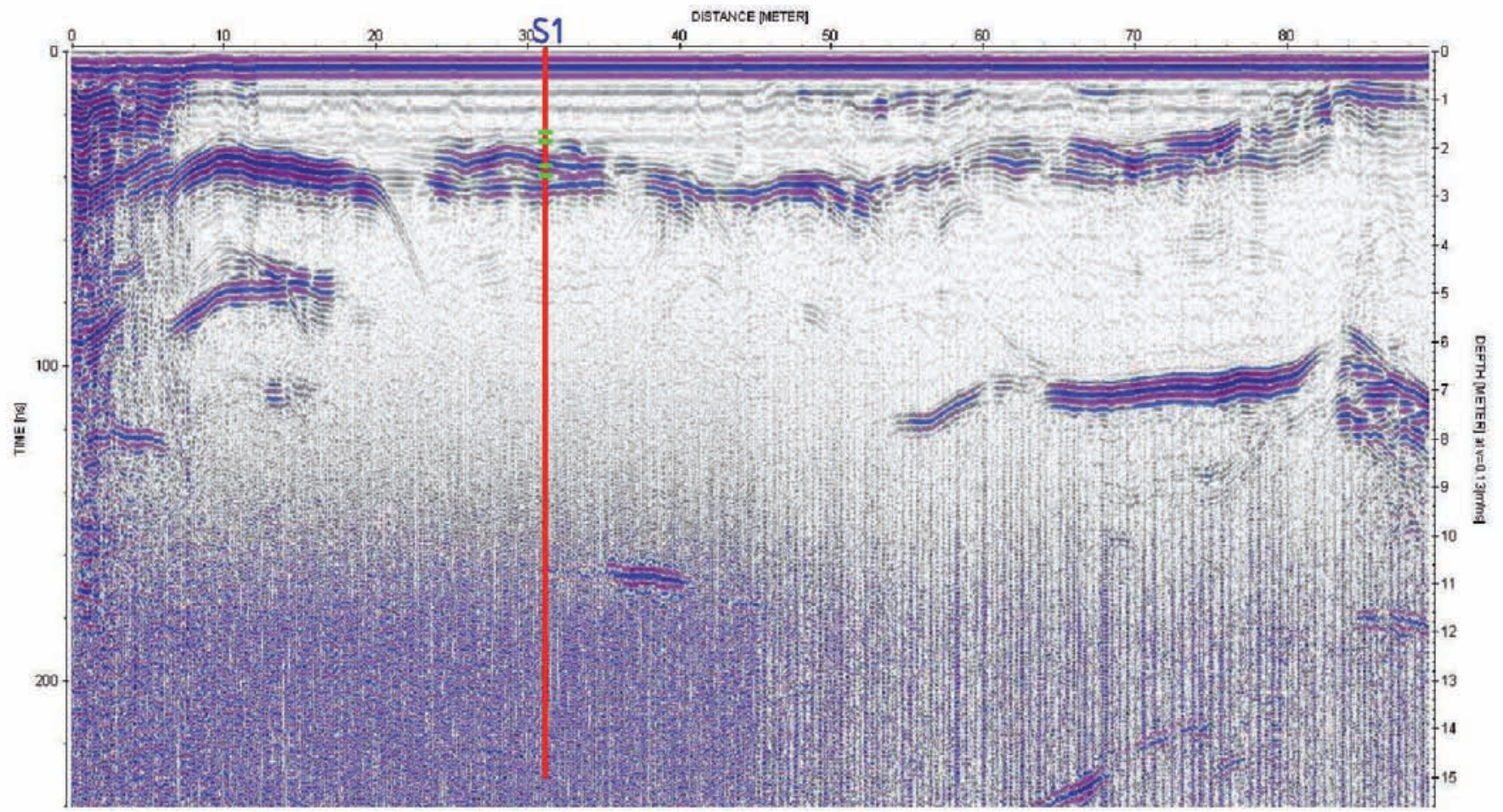

Figura 14. Perfil de georadar con antena de $250 \mathrm{MHz}$ en granito junto al sondeo estudiado.

Figure 14. GPR profile with $250 \mathrm{MHz}$ antenna in granite and studied drill.

nidas desde el punto de vista geológico como depósitos playeros con presencia de abundantes estructuras sedimentarias, fuertes cambios laterales de facies y discordancias que afectan a la explotación de este tipo de materiales. En el afloramiento estudiado se encontraba desde antaño una pequeña explotación de calcarenitas amarillas y blancas con mucha presencia de ripples de corriente y de karstificación. Una vez agotada esta explotación y antes de su cierre definitivo la empresa se dirigió al CTM para que se reali-

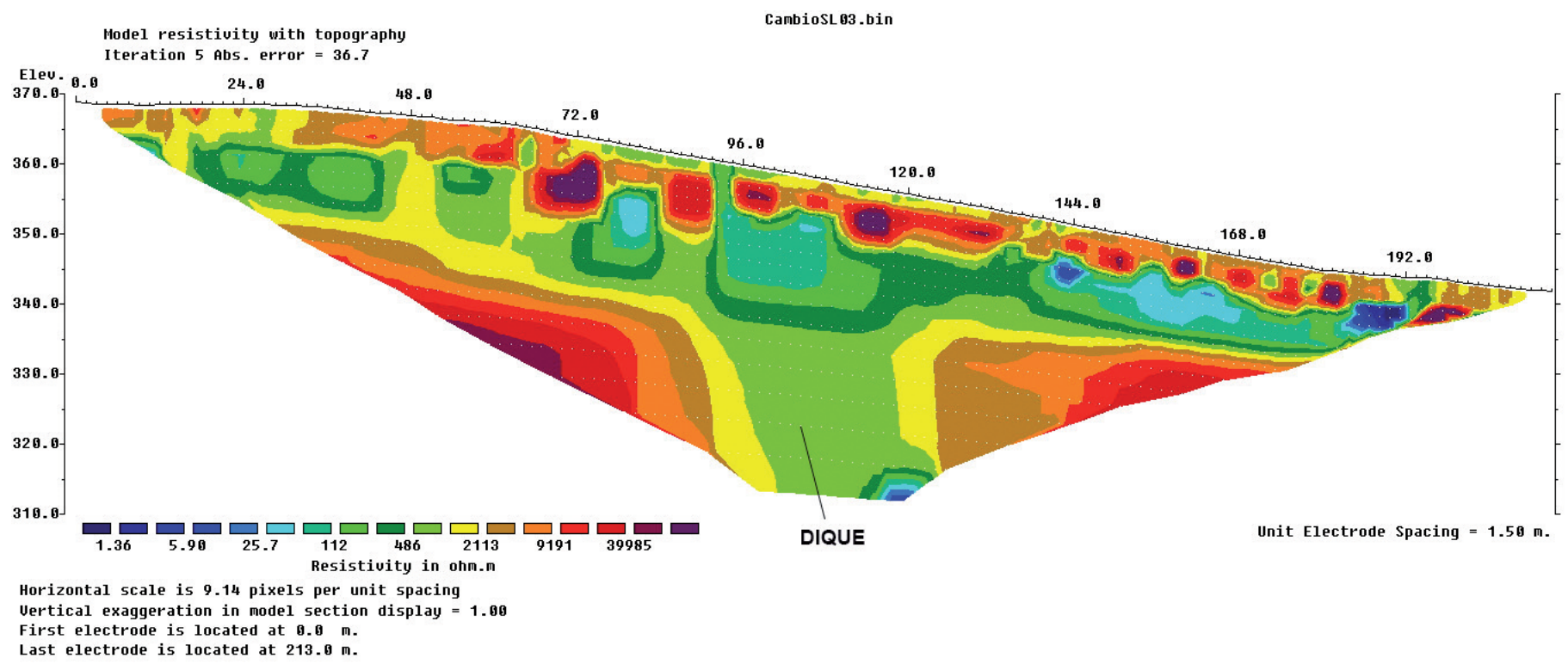

Figura 15. Perfil de tomografía en granito para identificar un dique de aplitas.

Figure 15. Tomography profile for identifying a granite aplite dike. 
zara una investigación minera de las cuadrículas cercanas para abrir otro frente. Tras las diferentes visitas realizadas se constató de la posible existencia de un ejemplo de ambiente sedimentario de playa-barrera, todo o en parte presente en esa zona, que posiblemente estaría sellado discordantemente por una potente capa de conglomerado poligénico y arcillas rojas con cantos de cuarcita y caliza con base erosiva. Esta es una formación continental que cubrió la zona formando una regresión marina ya que se pasa de sedimentación costera a continental que constituiría un primer relleno de esta área a finales de Langhiense.

Se diseñaron varias fases de investigación geofísica para obtener la estructura y tipo de material presente por debajo de esta capa de conglomerados.

Se han obtenido distintos perfiles que marcan la estructura existente en este afloramiento donde se encuentran conglomerados a base y techo de las calcarenitas explotables. Estos materiales son fácilmente karstificables provocando una situación importante para la empresa, ya que donde no existe la capa de conglomerados, como era la cantera antigua y zonas limítrofes, este proceso ha provocado una drástica caída en el aprovechamiento de este material, mientras que los conglomerados superiores han protegido de este proceso a la capa de calcarenitas suprayacente muy limpia de fracturas y con un aprovechamiento mayor donde solo las roturas del material provienen de la estratificación y de la laminación por los ripples existentes.

Este complejo de playa con barrera de acumulación posee hacia el E un detalle importante como es un canal de drenaje de la zona de playa con la barra, justo donde se encuentra un material muy trabajado y cementado.

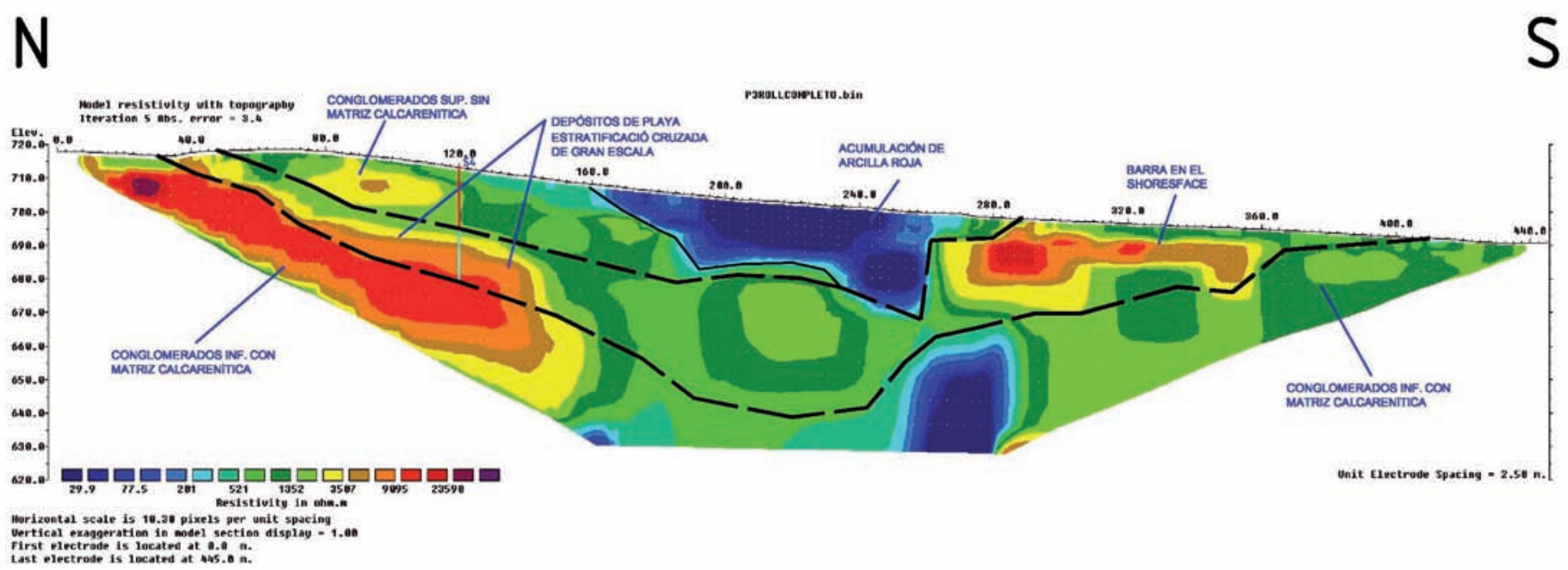

Figura 16. Perfil de tomografía realizado perpendicular a la estructura.

Figure 16. Tomography profile performed perpendicularly to the structure.

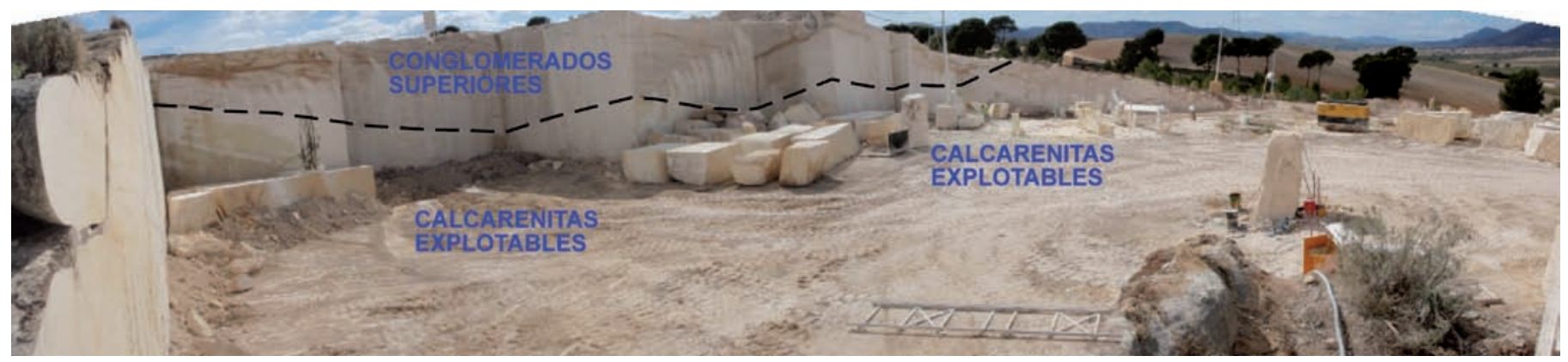

Figura 17. Imagen de la cantera con disposición de los materiales. Figure 17. Photograph of the quarry with the available materials. 
Espín de Gea, A., et al., 2017. Los métodos geofísicos como herramienta de mejora para... Boletín Geológico y Minero, 128 (2): $363-378$

\section{Conclusiones}

La incorporación por parte del CTM de las nuevas tecnologías en este sector de la roca ornamental, y más concretamente de métodos geofísicos para el desarrollo de proyectos de investigación minera, permite obtener gran cantidad de información de un yacimiento con unos costes limitados y de forma relativamente rápida.

Con este trabajo se pone de manifiesto que las técnicas geofísicas con las que se han realizado estos proyectos son una herramienta muy importante para conseguir información precisa y veraz de la estructura del yacimiento, calidad y volumen de reservas, permitiendo obtener una interpretación y cubicación, esencial para las labores futuras a realizar en estas explotaciones.

La combinación de varias técnicas geofísicas en la investigación de yacimientos de roca ornamental aporta gran calidad al resultado final, ya que cada una de ellas por separado presenta diferentes problemas que dificultan y pueden provocar algunas dudas en la interpretación, mientras que su utilización conjunta hace que se complementen, minimizando las zonas donde tienen problemas cada una por separado.

Las dificultades de aplicación también son importantes, ya que por ejemplo el georadar tiene muchos problemas con la presencia de capas de materiales finos, hecho muy abundante en nuestras zonas de estudio, mientras que es una técnica fácilmente aplicable, muy versátil en campo, ya que es de gran manejabilidad y con rapidez en la toma de decisiones "in situ" cuando se están realizando las medidas. La tomografía es una técnica difícil de encajar en las explotaciones de roca ornamental, debido a la longitud de los perfiles (máximo $355 \mathrm{~m}$ ) para alcanzar una profundidad de investigación de unos $70 \mathrm{~m}$, pero a su vez, es muy resolutiva y eficaz entre materiales con fuerte contraste de resistividades. La sísmica nos aporta información valiosa sobre la litología y el estado de alteración de los materiales del yacimiento, que podemos traducir en una clasificación de calidades del material, pero una dificultad es la presencia en las explotaciones de mucha maquinaria que provoca en las medidas la aparición de ruido.

Se ha podido constatar que su aplicación no está condicionada por el tipo de material, porque se han aplicado en diversos casos con resultados muy satisfactorios.

\section{Agradecimientos}

A las empresas del sector que han permitido la publicación de estos datos.

\section{Referencias}

Abbiss, C.P. (1981). Shear wave measurements of the elasticity of the ground. Geotechnique, Vol 31, no.1, p.91-104.

Beres, M., Luetsher, M. and Olivier, R. (2001). Integration of ground penetrating radar and microgravimetric methods to map shallow caves. Journal of Applied Geophysics 46, 249-262.

Collins, M. E., Cumb, M. and Hanninenc, P. (1994). Geoderma. Volume 61, Issues 1-2, Pages 1-15.

Conyers, L. B. (2004). Ground-penetrating Radar for Archaeology. Walnut Creek, CA, United States: AltaMira Press Ltd.

Daniels, D. J. (ed.) (2004). Ground Penetrating Radar (2nd $E d$.). Knoval (Institution of Engineering and Technology).

Espín de Gea, A. (2009). Analysis of karsts cave structure for the location of a new entrance using $3 D$ images obtained by ground penetrating radar. IWAGPR 2009.

Espín de Gea, A., Reyes, M., Gil, A. y Arévalo, L. (2014). Modelización geológica tridimensional del yacimiento de roca ornamental ubicado en Monte Coto parcelas K, $C$ y D. (Inédito).

Federación Española de la Piedra. (2013). Fondos propios.

Granda, A. y Cambero, J.C. (2012). Introducción al método de la Tomografía Eléctrica. International Geophysical Technology, S.L. (lgt).

Gómez López, R. (2008). Aplicación del radar de penetración en tierra (georadar) a la exploración no destructiva de yacimientos arqueológicos. http://maxwell.ugr.es/ ProyectoRaf/final2-babel-spanish.pdf.

IGME (2011). Panorama minero. Piedra natural. Disponible en: www.igme.es/internet/ PanoramaMinero/PMLin.htm

Menke, W. (1984). Geophysical Data Analysis: Discrete Inverse Theory. Academic Press, Orlando. USA.

Parasnis. D.S. (1997). Principles of Applied Geophysic. Chapman \& Hall.

Sharma, P.V. (2004). Enviromental and engineering geophysics. Cambridge University Press.

Telford,W.M., Geldart,L.P., Sheriff, R.E. (1990). Apllied geophysics. Second Edition. Cambridge University Press.

Trabajos Investigación Geológico Minera. Centro Tecnológico del Mármol. Proyectos propios.

Williams, P. W. (2008). The role of the epikarts in karst and cave hydrogeology: a review. International journal of Speleology 37, 1-10. Bologna (Italy). ISNN 0392-6672.

Recibido: diciembre 2015

Revisado: febrero 2016

Aceptado: abril 2016

Publicado: junio 2017 